\title{
Prevalence and risk factors of childhood allergic diseases in eight metropolitan cities in China: A multicenter study
}

Fei Li ${ }^{1,2,3 \dagger}$, Yingchun Zhou ${ }^{4 \dagger}$, Shenghui $\mathrm{Li}^{2,3}$, Fan Jiang ${ }^{1,2,3}$, Xingming Jin ${ }^{1,2,3}$, Chonghuai Yan ${ }^{2,3}$, Ying Tian ${ }^{2,3}$, Yiwen Zhang ${ }^{1,2,3}$, Shilu Tong ${ }^{5^{*}}$ and Xiaoming Shen ${ }^{1,2,3^{*}}$

\begin{abstract}
Background: Several studies conducted during the past two decades suggested increasing trend of childhood allergic diseases in China. However, few studies have provided detailed description of geographic variation and explored risk factors of these diseases. This study investigated the pattern and risk factors of asthma, allergic rhinitis and eczema in eight metropolitan cities in China.

Methods: We conducted a cross-sectional survey during November-December 2005 in eight metropolitan cities in China. A total of 23791 children aged 6-13 years participated in this survey. Questions from the standard questionnaire of the International Study of Asthma and Allergies in Children (ISAAC) were used to examine the pattern of current asthma, allergic rhinitis and eczema. Logistic regression analyses were performed to assess the risk factors for childhood allergies.

Results: The average prevalence of childhood asthma, allergic rhinitis and eczema across the eight cities was 3.3\% (95\% Confidence interval (Cl): 3.1\%, 3.6\%), 9.8\% (95\% Cl: 9.4\%, 10.2\%) and 5.5\% (95\% Cl: 5.2\%, 5.8\%), respectively. Factors related to lifestyle, mental health and socio-economic status were found to be associated with the prevalence of childhood allergies. These risk factors were unevenly distributed across cities and disproportionately affected the local prevalence.
\end{abstract}

Conclusions: There was apparent geographic variation of childhood allergies in China. Socio-environmental factors had strong impacts on the prevalence of childhood allergies; but these impacts differed across regions. Thus public health policies should specifically target at the local risk factors for each individual area.

\section{Background}

It is a significant and growing challenge to control and manage chronic allergic diseases worldwide. Approximately $20 \%$ of the world population suffers from allergic diseases which cause substantial health care burden [1]. For example, according to World Health Organization (WHO), 300 million people suffered from asthma and 255,000 died of asthma in 2005 [2].

\footnotetext{
*Correspondence: s.tong@qut.edu.au; xmshen@shsmu.edu.cn † Contributed equally

'Department of Developmental and Behavioral Pediatrics, Shanghai Institute of Pediatric Translational Medicine, Shanghai Children's Medical Centre, Shanghai Jiaotong University, School of Medicine, Shanghai, China ${ }^{5}$ School of Public Health and Institute of Health and Biomedical Innovation, Queensland University of Technology, Queensland, 4000, Australia Full list of author information is available at the end of the article
}

Around the year 2000, the International Study of Asthma and Allergies in Children (ISAAC) was conducted across different regions of the world, which indicated that China's allergic problems could not be ignored [3]. However, their results only covered two cities in mainland China. In 1990 and 2000, two national surveys were conducted and showed that the prevalence of asthma among children 0-14 years old was $1 \%$ and $1.97 \%$ [4], respectively, suggesting an increasing trend of asthma. These implied that the socioenvironmental changes might directly or indirectly affect the prevalence rates of asthma and allergies. However, few data are available on the national pattern of prevalence and associated risk factors of childhood allergies in China.

\section{Biomed Central}


A national survey on children's sleep was conducted in eight Chinese cities in 2005 [5]. Since this survey covered a range of childhood illnesses including asthma, allergic rhinitis and eczema, it offers a unique opportunity to examine the national trends of children's allergic diseases and their risk factors in China.

\section{Methods \\ Sample}

A cross-sectional survey was undertaken among children aged 6-13 years old in eight Chinese cities during November and December 2005, using a cluster-stratified sampling method. The eight cities included Shanghai, Guangzhou, Xi'an, Wuhan, Harbin, Chengdu, Hohhot and Urumqi. These were capital cities of provinces located in four different regions (Additional File 1, Figure S1) [6]. The reason for choosing capital cities was that, in China, these capital cities generally have high levels of health resources and public awareness about childhood allergic diseases, and doctors generally follow the national standard of clinical diagnosis. Therefore, the reporting of childhood allergic conditions and many of their risk factors would be more reliable. Three to ten districts were randomly selected from each city, and 1-2 elementary schools from each district. The number of districts was determined by the size of the city and the number of schools determined by the size of the district. The final sample comprised thirty districts and 42 schools in urban areas, and 9 districts and 13 schools in suburban areas. Of 23,791 children from 6 grades of the chosen schools, 22,018 (the response rate: 92.5\%) participated in the survey and returned completed questionnaires (Additional File 2, Figure S2).

\section{Data collection}

The survey was conducted by the Key Laboratory of Children's Environmental Health in Shanghai, China. A national steering committee, comprised highly experienced paediatricians and epidemiologists from each survey centre, was established; a uniform research protocol was used by each centre; and formal training for the survey interviewers was provided. Each questionnaire was completed by a parent or guardian of a child after an informed consent form was signed. To ensure credibility and accuracy of the survey, we randomly selected 239 children for re-evaluation of responses one month after the first interview. 123 questionnaires for testing parallel consistency (ie, the consistency between the results when father and mother complete the same survey at the same time) and 116 questionnaires for assessing test-retest reliability were completed. The internal consistency of overall questionnaire was good (Cronbach's alpha's coefficient: $0.73)$. The parallel consistency between mother and father was represented by intro-class correlation coefficient (ICC: 0.89 ), and the test-retest reliability was also high (ICC: 0.85 ). To allow for comparison with results in the literature, we used key questions from the standard ISAAC questionnaire to examine the prevalences of current asthma, allergic rhinitis and eczema. The questions regarding asthma, allergic rhinitis and eczema were: "Has the child had asthma in the past 12 months?" (Yes or no), "Has the child had allergic rhinitis in the past 12 months?" (Yes or no) and "Has the child had eczema in the past 12 months?" (Yes or no). Data on a wide range of variables concerning demographic, lifestyle, mental health, socioeconomic status (SES) and health conditions were also collected: including child's age, gender, mode of delivery, breast feeding, diagnosed obesity, diagnosed gastroesophageal reflux (GER), carbonated drinks intake, computer use for amusements, schoolwork burden, mood, environmental tobacco exposure, parental alcohol abuse, parental smoking, maternal diagnosed depression during pregnancy and postpartum period, parental diagnosed depression, family structure, diagnosed childhood attention deficit hyperactivity disorder (ADHD), parental education level, household income per capita, resident area per capita, family size, common cold, diagnosed recurrent otitis media, snoring, sleep disordered breathing, parental snoring, parental sleep disordered breathing, sleep duration, and parental age at child's birth.

\section{Ethics statement}

The ethical application of this study was approved by the local institutional review board at each research site, which included institutional review board of Shanghai Jiaotong University School of Medicine, Sichuan University West China Center of Medical Sciences, Sun Yat Sen University Medical School, Huazhong University of Science and Technology Tongji Medical University, Xi'an Jiaotong University College of Medicine, Harbin Medical University, Inner Mongolia Medical College and Xinjiang Medical University. We had obtained the written informed consent from all participants involved in our study.

\section{Statistical analysis}

The prevalence rates of asthma, allergic rhinitis and eczema of each city were adjusted for age and sex [7], when compared across cities. Differences among cities were examined using chi-square tests for categorical variables and analyses of variance for continuous variables (significance level $\mathrm{p}=0.05$ for two tails).

Logistic regression analyses were performed to assess the risk factors of allergies such as demographic variables, life styles, mental health and SES. Interaction 
terms of specific variables were also considered. The regression model adopted a forward stepwise elimination procedure, with probabilities for variable entry and removal of 0.05 and 0.10 , respectively. Statistical tests of the regression estimates or odds ratios (OR) were based on Wald statistics. All analyses were conducted using SPSS version 13.0.

\section{Results}

\section{Characteristics of study subjects}

The mean age of the sample was 9.18 years $(S D=1.75)$, with 10,366 male students $(49.6 \%)$ and 10,548 female students $(50.4 \%)$. Of the 22018 children, the vast majority $(20,719 ; 94.1 \%)$ came from the Han ethnic group, with the remaining children $(1,299 ; 5.9 \%)$ from other ethnic minorities.

There was marked difference in some characteristics across cities. (Additional File 3, Table S1). For example, compared with those from other regions, participants from the eastern region (including Shanghai and Guangzhou) appeared to have higher levels of parental education and household income per capita, higher diagnosed obesity rates and more frequent computer use for amusement, lower vaginal delivery and breast feeding rates.

\section{Patterns of allergic diseases}

Table 1 shows both crude and adjusted (adjusted for age and sex) prevalences of three allergic diseases of each city. Figure 1 reveals the crude prevalence by gender across cities. The average prevalence of asthma, allergic rhinitis and eczema across eight cities was 3.3\% (3.1, $3.6), 9.8 \%(9.4,10 \cdot 2)$ and $5.5 \%(5 \cdot 2,5 \cdot 8)$, respectively. There were statistically significant differences in the

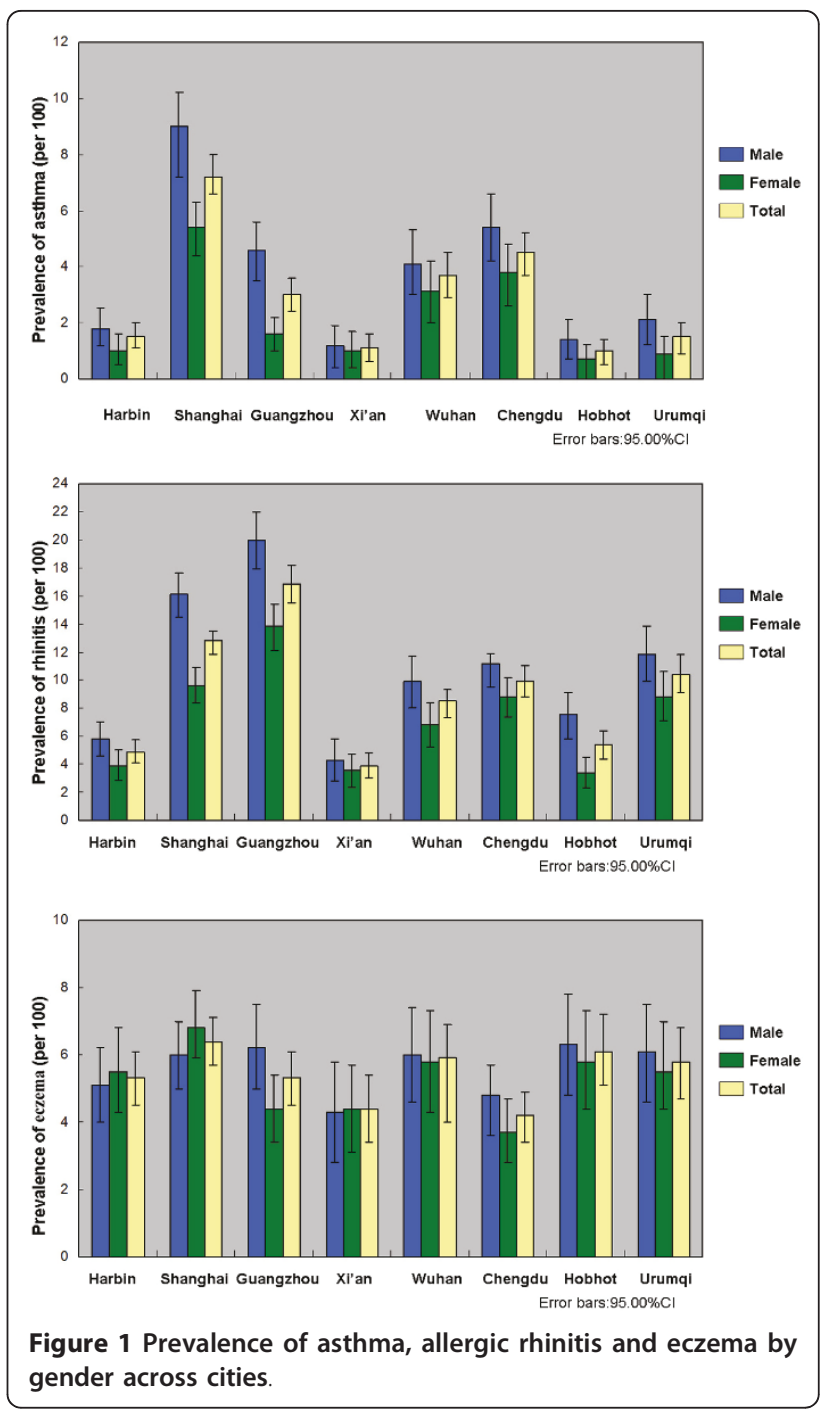

Table 1 Crude and adjusted* prevalence rates (\%) of three allergic diseases

\begin{tabular}{|c|c|c|c|c|c|c|c|}
\hline & \multirow[b]{2}{*}{ Number } & \multicolumn{2}{|c|}{ Asthma } & \multicolumn{2}{|c|}{ Allergic rhinitis } & \multicolumn{2}{|c|}{ Eczema } \\
\hline & & $\begin{array}{l}\text { Crude } \\
\text { prevalence (\%) } \\
(95 \% \mathrm{Cl})\end{array}$ & $\begin{array}{l}\text { Adjusted } \\
\text { prevalence (\%) } \\
(95 \% \mathrm{Cl})\end{array}$ & $\begin{array}{l}\text { Crude } \\
\text { prevalence (\%) } \\
(95 \% \mathrm{Cl})\end{array}$ & $\begin{array}{l}\text { Adjusted } \\
\text { prevalence (\%) } \\
(95 \% \mathrm{Cl})\end{array}$ & $\begin{array}{l}\text { Crude } \\
\text { prevalence (\%) } \\
(95 \% \mathrm{CI})\end{array}$ & $\begin{array}{l}\text { Adjusted } \\
\text { prevalence (\%) } \\
(95 \% \mathrm{Cl})\end{array}$ \\
\hline Total & 22,009 & $3 \cdot 3(3 \cdot 1-3 \cdot 6)$ & $3 \cdot 3(3 \cdot 1-3 \cdot 6)$ & $9.8(9.4-10.2)$ & $9.8(9.4-10 \cdot 2)$ & $5 \cdot 5(5 \cdot 2-5 \cdot 8)$ & $5 \cdot 5(5 \cdot 2-5 \cdot 8)$ \\
\hline Harbin(4) & 2,900 & $1.4(1.0-1.9)$ & $1 \cdot 7(1 \cdot 0-2 \cdot 4)$ & $4.9(4 \cdot 1-5 \cdot 7)$ & $4.9(3.9-5.8)$ & $5 \cdot 3(4 \cdot 5-6 \cdot 1)$ & $4.7(3.9-5 \cdot 5)$ \\
\hline $\begin{array}{l}\text { Shanghai } \\
\text { (10) }\end{array}$ & 4,395 & $7 \cdot 2(6.4-8.0)$ & $7.0(6.0-7.9)$ & $12.9(11.9-13.9)$ & $13 \cdot 1(11 \cdot 7-14 \cdot 5)$ & $6 \cdot 4(5 \cdot 7-7 \cdot 1)$ & $6.5(5.4-7.5)$ \\
\hline $\begin{array}{l}\text { Guangzhou } \\
\text { (4) }\end{array}$ & 3,094 & $3.0(2.4-3.6)$ & $3.0(2 \cdot 4-3.6)$ & $16 \cdot 7(15.4-18.0)$ & $16 \cdot 8(15 \cdot 3-18 \cdot 2)$ & $5 \cdot 3(4 \cdot 5-6 \cdot 1)$ & $5 \cdot 4(4 \cdot 6-6 \cdot 2)$ \\
\hline X'an(3) & 1,653 & $1 \cdot 1(0.6-1.6)$ & $1 \cdot 1(0.6-1 \cdot 7)$ & $3.9(3.0-4 \cdot 8)$ & $3.9(2.9-4.9)$ & $4.4(3.4-5.4)$ & $4.4(3.4-5.4)$ \\
\hline Wuhan(4) & 2,061 & $3 \cdot 6(2 \cdot 8-4 \cdot 4)$ & $3.5(2.7-4.3)$ & $8.5(7 \cdot 3-9.7)$ & $8.3(7 \cdot 1-9 \cdot 6)$ & $6 \cdot 0(5 \cdot 0-7 \cdot 0)$ & $6 \cdot 1(5 \cdot 0-7 \cdot 2)$ \\
\hline Chengdu(5) & 2,848 & $4.5(3 \cdot 8-5 \cdot 3)$ & $4.6(3.8-5.4)$ & $9.9(8.8-11.0)$ & $10 \cdot 1(8.9-11 \cdot 2)$ & $4.2(3.5-4.9)$ & $4.3(3 \cdot 5-5 \cdot 1)$ \\
\hline Hohhot(5) & 2,025 & $1.0(0.6-1.5)$ & $0.9(0.5-1.4)$ & $5 \cdot 4(4 \cdot 4-6 \cdot 4)$ & $4.5(3 \cdot 6-5 \cdot 4)$ & $6 \cdot 2(5 \cdot 2-7 \cdot 2)$ & $6.4(4.9-7.9)$ \\
\hline Urumqi(4) & 2,033 & $1 \cdot 5(1 \cdot 0-2 \cdot 1)$ & $1.6(1 \cdot 0-2 \cdot 1)$ & $10 \cdot 3(9 \cdot 0-11 \cdot 6)$ & $10 \cdot 1(8 \cdot 6-11 \cdot 5)$ & $5.9(4.9-6.9)$ & $5.9(4 \cdot 8-6 \cdot 9)$ \\
\hline$* * P$ & & $<0.001$ & $<0.001$ & $<0.001$ & $<0.001$ & $<0.001$ & $<0.001$ \\
\hline
\end{tabular}

Numbers of centers is indicated in brackets next to city name.*Adjusted to the age and gender distribution of the total sample. ${ }^{* *} \mathrm{P}$-value for difference between sites. 
prevalences of asthma and allergic rhinitis $(\mathrm{p}<0.001)$ among the eight cities. There was no statistically significant difference in the geographic pattern of eczema.

\section{Risk factors of allergic diseases}

Table 2 shows the predictors of asthma, allergic rhinitis and eczema at the national level. In general, these allergies have many risk factors including biological (eg, mode of delivery, breast feeding, and sleep-disordered breathing); lifestyle (eg, diagnosed obesity, carbonated drinks intake, and frequent computer use as amusement); mental health (eg, diagnosed prepartum and postpartum depression, diagnosed childhood ADHD, and family structure); SES (eg, paternal education level, maternal education level, and household income per capita) and health conditions (eg, common cold, paternal snoring history, diagnosed recurrent otitis media, and diagnosed GER and snoring). Three allergic diseases seemed to share some common risk factors including residential city, diagnosed childhood ADHD, maternal education level, common cold, paternal snoring, and sleep-disordered breathing. Additionally,

Table 2 Multiple logistic regression of risk factors for three allergic diseases

\begin{tabular}{|c|c|c|c|c|}
\hline \multicolumn{2}{|l|}{ Variables } & \multirow{2}{*}{$\begin{array}{c}\begin{array}{c}\text { Asthma } \\
\text { OR(95\% Cl) }\end{array} \\
1 \cdot 5(1 \cdot 3-1 \cdot 8)\end{array}$} & \multirow{2}{*}{$\begin{array}{c}\begin{array}{c}\text { Allergic } \\
\text { rhinitis } \\
\text { OR(95\% Cl) }\end{array} \\
1.4(1.2-1.5)\end{array}$} & \multirow[t]{2}{*}{$\begin{array}{c}\text { Eczema } \\
\text { OR(95\% Cl) }\end{array}$} \\
\hline Gender & Male & & & \\
\hline $\mathrm{Age}^{3}$ & & & $1 \cdot 1(1 \cdot 0-1 \cdot 2)$ & \\
\hline Diagnosed GER & Yes & $2 \cdot 3(1 \cdot 2-4 \cdot 3)$ & & \\
\hline Diagnosed obesity & Yes & $1 \cdot 7(1 \cdot 1-2 \cdot 7)$ & & $1.5(1 \cdot 0-2 \cdot 3)$ \\
\hline \multirow[t]{2}{*}{ Mode of delivery } & Caesarean (Ref.) & & & \\
\hline & Vaginal & $0.8(0.7-0.9)$ & & \\
\hline Carbonated drinks intake & $>=5$ times/week & $1 \cdot 8(1 \cdot 3-2 \cdot 6)$ & & \\
\hline Exclusive breast feeding & $>=4$ months & & $0.8(0.7-0.9)$ & \\
\hline Computer use as amusements & $>=5$ times/week & & & $1 \cdot 3(1 \cdot 1-1 \cdot 6)$ \\
\hline Diagnosed childhood ADHD & Yes & $1 \cdot 4(1 \cdot 0-2 \cdot 0)$ & $1 \cdot 7(1 \cdot 4-2 \cdot 1)$ & $1 \cdot 5(1 \cdot 2-2 \cdot 0)$ \\
\hline Diagnosed prepartum and postpartum depression & Yes & & & $1 \cdot 7(1 \cdot 3-2 \cdot 4)$ \\
\hline \multirow[t]{2}{*}{ Family structure } & Single-parent and extended family (Ref.) & & & \\
\hline & Nuclear family & $0.8(0.7-1.0)$ & & \\
\hline Household income per capita & $>=1500 \mathrm{RMB} /$ month & $1 \cdot 3(1 \cdot 1-1 \cdot 7)$ & $1 \cdot 2(1 \cdot 1-1 \cdot 4)$ & \\
\hline Maternal education level & $>=$ High school graduate & $1.5(1 \cdot 2-1 \cdot 8)$ & $1 \cdot 2(1 \cdot 1-1 \cdot 4)$ & $1 \cdot 3(1 \cdot 2-1 \cdot 6)$ \\
\hline Paternal education level & $>=$ High school graduate & & $1 \cdot 3(1 \cdot 1-1 \cdot 4)$ & \\
\hline $\begin{array}{l}\text { Maternal education level *Household income per } \\
\text { capita }\end{array}$ & $\begin{array}{l}>=\text { High school graduate and }>= \\
\text { 1500RMB } / \text { month }\end{array}$ & $1.6(1.1,2.4)$ & & \\
\hline Common cold & $>5$ times/year & $3 \cdot 2(2 \cdot 7-3 \cdot 8)$ & $2 \cdot 3(2 \cdot 1-2 \cdot 6)$ & $1 \cdot 8(1 \cdot 5-2 \cdot 1)$ \\
\hline Snoring & Yes & & $1.5(1 \cdot 3-1 \cdot 7)$ & \\
\hline Sleep-disordered breathing & Yes & $2 \cdot 3(1 \cdot 6-3 \cdot 1)$ & $2 \cdot 3(1 \cdot 9-2 \cdot 9)$ & $1.5(1 \cdot 1-2 \cdot 0)$ \\
\hline Paternal snoring & Yes & $1 \cdot 2(1 \cdot 0-1 \cdot 5)$ & $1 \cdot 2(1 \cdot 1-1 \cdot 3)$ & $1 \cdot 2(1 \cdot 0-1 \cdot 4)$ \\
\hline Parental age at child's birth & & $1 \cdot 2(1 \cdot 1-1 \cdot 3)$ & $1 \cdot 1(1 \cdot 0-1 \cdot 3)$ & \\
\hline Diagnosed recurrent otitis media & Yes & & $2 \cdot 1(1 \cdot 8-2 \cdot 6)$ & $1.9(1 \cdot 5-2 \cdot 4)$ \\
\hline Diagnosed asthma & Yes & & $4 \cdot 3(3 \cdot 6-5 \cdot 2)$ & $2 \cdot 7(2 \cdot 2-3 \cdot 4)$ \\
\hline Diagnosed allergic rhinitis & Yes & $3 \cdot 2(2 \cdot 7-3 \cdot 6)$ & & $1 \cdot 8(1 \cdot 5-2 \cdot 1)$ \\
\hline Diagnosed eczema & Yes & $2 \cdot 9(2 \cdot 3-3 \cdot 7)$ & $1 \cdot 8(1 \cdot 5-2 \cdot 2)$ & \\
\hline Common cold*Snoring & Yes & & $1.3(1.0,1.8)$ & \\
\hline \multirow[t]{8}{*}{ Site } & Urumqi (Ref.) & & & \\
\hline & Shanghai & $4 \cdot 4(2 \cdot 9-6 \cdot 6)$ & $1 \cdot 0(0 \cdot 9-1 \cdot 3)$ & $0 \cdot 9(0 \cdot 7-1 \cdot 2)$ \\
\hline & Guangzhou & $1 \cdot 4(0 \cdot 9-2 \cdot 2)$ & $1 \cdot 6(1 \cdot 3-1.9)$ & $0 \cdot 8(0 \cdot 6-1 \cdot 1)$ \\
\hline & Xi'an & $1 \cdot 4(3 \cdot 7-2 \cdot 6)$ & $0.4(0.3-0.6)$ & $0 \cdot 9(0 \cdot 6-1 \cdot 2)$ \\
\hline & Wuhan & $2 \cdot 7(1 \cdot 7-4 \cdot 4)$ & $0 \cdot 9(0 \cdot 7-1 \cdot 1)$ & $1 \cdot 1(0 \cdot 8-1 \cdot 5)$ \\
\hline & Chengdu & $3 \cdot 2(2 \cdot 0-4 \cdot 9)$ & $0 \cdot 8(0 \cdot 7-1 \cdot 0)$ & $0.6(0.5-0.8)$ \\
\hline & Harbin & $1 \cdot 4(0 \cdot 8-2 \cdot 3)$ & $0.5(0.4-0.6)$ & $1 \cdot 0(0 \cdot 7-1 \cdot 3)$ \\
\hline & Hohhot & $0 \cdot 9(0 \cdot 5-1 \cdot 7)$ & $0.6(0.4-0.7)$ & $1 \cdot 3(1 \cdot 0-1 \cdot 7)$ \\
\hline
\end{tabular}

Values highlighted in bold indicate OR within $95 \% \mathrm{Cl}$ and statistically significant at $\mathrm{P}<0.05$. 
asthma and allergic rhinitis even shared more common risk factors such as gender, household income per capita, and paternal age at child's birth.

Tables 3, 4 and 5 show the specific risk factors of asthma, allergic rhinitis and eczema in the eight cities, respectively. Some cities shared common risk factors. For example, SES factors such as higher parental education level and higher household income per capita were associated with higher prevalences in Shanghai, Guangzhou, Wuhan and Chengdu, which are four biggest and affluent cities in this study. On the other hand, different cities had different spectrums of risk factors. For example, mental health factors were strongly associated with asthma in Wuhan (eg, OR $=3.45$, (95\% CI: 1.04, 11.45) for diagnosed maternal depression), while life style factors such as diagnosed GER was a significant risk factor of asthma in Chengdu (OR = 14.6, (95\% CI: 12.1, 41.7), and $5 \cdot 3$, (95\% CI: $1 \cdot 2,6 \cdot 7)$, respectively). Risk factors of allergic rhinitis (Table 4) and eczema (Table 5) show similar patterns as those of asthma (Table 3).

Table 3 Local level risk factors of asthma: results of multiple logistic regression analysis

\begin{tabular}{|c|c|c|c|c|c|c|c|c|c|}
\hline Variables & & $\begin{array}{c}\text { Shanghai } \\
\text { OR } \\
(95 \% \mathrm{Cl})\end{array}$ & $\begin{array}{c}\text { Guangzhou } \\
\text { OR } \\
(95 \% \mathrm{Cl})\end{array}$ & $\begin{array}{l}\text { Xi'an OR } \\
(95 \% \mathrm{Cl})\end{array}$ & $\begin{array}{c}\text { Wuhan } \\
\text { OR } \\
(95 \% \mathrm{Cl})\end{array}$ & $\begin{array}{c}\text { Chengdu } \\
\text { OR } \\
(95 \% \mathrm{Cl})\end{array}$ & $\begin{array}{l}\text { Harbin OR } \\
(95 \% \mathrm{Cl})\end{array}$ & $\begin{array}{c}\text { Hohhot } \\
\text { OR } \\
(95 \% \mathrm{Cl})\end{array}$ & $\begin{array}{c}\text { Urumqi } \\
\text { OR } \\
(95 \% \mathrm{Cl})\end{array}$ \\
\hline Gender & Male & $\begin{array}{c}1.5 \\
(1.1-1.9)\end{array}$ & $\begin{array}{c}2 \cdot 3 \\
(1 \cdot 4-3 \cdot 8)\end{array}$ & & & & & & \\
\hline Diagnosed GER & Yes & & & & & $\begin{array}{c}14 \cdot 6 \\
(12 \cdot 1-41 \cdot 7)\end{array}$ & & $\begin{array}{c}10 \cdot 6 \\
(23 \cdot 4-48 \cdot 5)\end{array}$ & \\
\hline Diagnosed Obesity & Yes & & & & & & $\begin{array}{c}14 \cdot 1 \\
(5 \cdot 0-40 \cdot 1)\end{array}$ & $\begin{array}{c}7.9 \\
(1.7-35.8)\end{array}$ & $\begin{array}{c}7 \cdot 7 \\
(2 \cdot 2-27 \cdot 2)\end{array}$ \\
\hline $\begin{array}{l}\text { Carbonated drinks } \\
\text { intake }\end{array}$ & $>=5$ times/week & & & & & $\begin{array}{c}5 \cdot 3 \\
(1 \cdot 2-6 \cdot 7)\end{array}$ & & & \\
\hline $\begin{array}{l}\text { Maternal diagnosed } \\
\text { depression }\end{array}$ & Yes & & & & $\begin{array}{c}5.5 \\
(1.4-11.4)\end{array}$ & & & & \\
\hline $\begin{array}{l}\text { Diagnosed } \\
\text { prepartum and } \\
\text { postpartum } \\
\text { depression }\end{array}$ & Yes & $\begin{array}{c}2 \cdot 4 \\
(1 \cdot 2-4 \cdot 9)\end{array}$ & & & & & & & \\
\hline \multirow[t]{2}{*}{ Family structure } & $\begin{array}{l}\text { Single-parent and } \\
\text { extended family } \\
\text { (Ref.) }\end{array}$ & & & & & & & & \\
\hline & Nuclear family & & & & & $\begin{array}{c}3 \cdot 2 \\
(1 \cdot 8-8 \cdot 5)\end{array}$ & & & \\
\hline $\begin{array}{l}\text { Household income } \\
\text { per capita }\end{array}$ & $\begin{array}{l}>=1500 \mathrm{RMB} / \\
\text { month }\end{array}$ & & $\begin{array}{c}2 \cdot 2 \\
(1 \cdot 1-1 \cdot 7)\end{array}$ & & $\begin{array}{c}1 \cdot 4 \\
(1 \cdot 1-3 \cdot 6)\end{array}$ & & & & \\
\hline $\begin{array}{l}\text { Maternal education } \\
\text { level }\end{array}$ & $\begin{array}{l}>=\text { High school } \\
\text { graduate }\end{array}$ & $\begin{array}{c}1.5 \\
(1 \cdot 2-2 \cdot 0)\end{array}$ & $\begin{array}{c}1 \cdot 8 \\
(1 \cdot 1-3 \cdot 0)\end{array}$ & & & & & & \\
\hline $\begin{array}{l}\text { Paternal education } \\
\text { level }\end{array}$ & $\begin{array}{l}>=\text { High school } \\
\text { graduate }\end{array}$ & & & & & $\begin{array}{c}2.8 \\
(1.8-4 \cdot 5)\end{array}$ & & & \\
\hline $\begin{array}{l}\text { Maternal education } \\
\text { level *Household } \\
\text { income per capita }\end{array}$ & $\begin{array}{l}>=\text { High school } \\
\text { graduate and }>= \\
\text { 1500RMB } / \text { month }\end{array}$ & & $\begin{array}{c}1.6 \\
(1.0,2.7)\end{array}$ & & & & & & \\
\hline $\begin{array}{l}\text { Paternal education } \\
\text { level *Household } \\
\text { income per capita }\end{array}$ & $\begin{array}{l}>=\text { High school } \\
\text { graduate and }>= \\
\text { 1500RMB } / \text { month }\end{array}$ & & & & $\begin{array}{c}2.3 \\
(1.2,4.6)\end{array}$ & & & & \\
\hline Common cold & $>5$ times/year & $\begin{array}{c}2 \cdot 6 \\
(1 \cdot 9-3 \cdot 3)\end{array}$ & $\begin{array}{c}3 \cdot 8 \\
(2 \cdot 3-6 \cdot 1)\end{array}$ & $\begin{array}{c}7 \cdot 1 \\
(2 \cdot 6-19 \cdot 2)\end{array}$ & $\begin{array}{c}5 \cdot 8 \\
(3 \cdot 7-12 \cdot 3)\end{array}$ & $\begin{array}{c}2 \cdot 7 \\
(1 \cdot 8-4 \cdot 2)\end{array}$ & $\begin{array}{c}3.9 \\
(2 \cdot 0-7 \cdot 8)\end{array}$ & & $\begin{array}{c}3.0 \\
(1 \cdot 3-6 \cdot 7)\end{array}$ \\
\hline $\begin{array}{l}\text { Sleep-disordered } \\
\text { breathing }\end{array}$ & Yes & $\begin{array}{c}1.9 \\
(1 \cdot 1-3 \cdot 4)\end{array}$ & & & & $\begin{array}{c}3.9 \\
(1.8-8 \cdot 5)\end{array}$ & & $\begin{array}{c}5 \cdot 3 \\
(1 \cdot 6-17 \cdot 1)\end{array}$ & $\begin{array}{c}4 \cdot 1 \\
(1.5-11 \cdot 4)\end{array}$ \\
\hline Maternal snoring & Yes & & & & & & & & $\begin{array}{c}3.3 \\
(1 \cdot 2-8 \cdot 7)\end{array}$ \\
\hline $\begin{array}{l}\text { Paternal age at } \\
\text { child's birth }\end{array}$ & & $\begin{array}{c}1 \cdot 0 \\
(1 \cdot 0-1 \cdot 1)\end{array}$ & & & & & & & \\
\hline $\begin{array}{l}\text { Diagnosed allergic } \\
\text { rhinitis }\end{array}$ & Yes & $\begin{array}{c}5 \cdot 3 \\
(4 \cdot 0-6 \cdot 9)\end{array}$ & $\begin{array}{c}4 \cdot 4 \\
(2 \cdot 7-7 \cdot 1)\end{array}$ & & $\begin{array}{c}4 \cdot 6 \\
(3 \cdot 0-10 \cdot 4)\end{array}$ & $\begin{array}{c}5.7 \\
(3.7-8.9)\end{array}$ & $\begin{array}{c}3 \cdot 8 \\
(1 \cdot 6-9 \cdot 1)\end{array}$ & $\begin{array}{c}5 \cdot 4 \\
(1 \cdot 8-16 \cdot 2)\end{array}$ & $\begin{array}{c}2.8 \\
(1 \cdot 1-6 \cdot 8)\end{array}$ \\
\hline Diagnosed eczema & Yes & $\begin{array}{c}3 \cdot 2 \\
(2 \cdot 2-4 \cdot 5)\end{array}$ & $\begin{array}{c}2.9 \\
(1.5-5 \cdot 6)\end{array}$ & & $\begin{array}{c}2 \cdot 4 \\
(1 \cdot 1-5 \cdot 2)\end{array}$ & $\begin{array}{c}2 \cdot 4 \\
(1 \cdot 2-4 \cdot 7)\end{array}$ & $\begin{array}{c}4 \cdot 1 \\
(1 \cdot 8-9 \cdot 2)\end{array}$ & & $\begin{array}{c}3 \cdot 1 \\
(1 \cdot 1-8 \cdot 2)\end{array}$ \\
\hline
\end{tabular}

Values highlighted in bold indicate $\mathrm{OR}$ within $95 \% \mathrm{Cl}$ and statistically significant at $\mathrm{P}<0.05$. 
Table 4 Local level risk factors of allergic rhinitis: results of multiple logistic regression analysis

\begin{tabular}{|c|c|c|c|c|c|c|c|c|c|}
\hline Variables & & $\begin{array}{c}\text { Shanghai } \\
\text { OR } \\
(95 \% \mathrm{Cl})\end{array}$ & $\begin{array}{c}\text { Guangzhou } \\
\text { OR } \\
(95 \% \mathrm{Cl})\end{array}$ & $\begin{array}{l}\text { Xi'an OR } \\
(95 \% \mathrm{Cl})\end{array}$ & $\begin{array}{c}\text { Wuhan } \\
\text { OR } \\
(95 \% \mathrm{Cl})\end{array}$ & $\begin{array}{c}\text { Chengdu } \\
\text { OR } \\
(95 \% \mathrm{Cl})\end{array}$ & $\begin{array}{c}\text { Harbin OR } \\
(95 \% \mathrm{Cl})\end{array}$ & $\begin{array}{c}\text { Hohhot } \\
\text { OR } \\
(95 \% \mathrm{Cl})\end{array}$ & $\begin{array}{l}\text { Urumqi } \\
\text { OR } \\
(95 \% \mathrm{Cl})\end{array}$ \\
\hline \multirow[t]{2}{*}{ Gender } & Female (Ref.) & & & & & & & & \\
\hline & Male & $\begin{array}{c}1.5 \\
(1.3-1.9)\end{array}$ & $\begin{array}{c}1.3 \\
(1 \cdot 1-1 \cdot 6)\end{array}$ & & $\begin{array}{c}1.5 \\
(1.0-2 \cdot 2)\end{array}$ & $\begin{array}{c}2 \cdot 3 \\
(1 \cdot 7-3 \cdot 1)\end{array}$ & & $\begin{array}{c}2 \cdot 4 \\
(1 \cdot 5-3 \cdot 1)\end{array}$ & \\
\hline Age & & $\begin{array}{c}1 \cdot 2 \\
(1 \cdot 1-1 \cdot 2)\end{array}$ & & & $\begin{array}{c}1 \cdot 2 \\
(1 \cdot 1-1 \cdot 3)\end{array}$ & & & $\begin{array}{c}1 \cdot 3 \\
(1 \cdot 1-1 \cdot 4)\end{array}$ & $\begin{array}{c}1 \cdot 2 \\
(1 \cdot 1-1 \cdot 3)\end{array}$ \\
\hline $\begin{array}{l}\text { Exclusive breast } \\
\text { feeding }\end{array}$ & $>=4$ months & $\begin{array}{c}0.8 \\
(0.6-0.9)\end{array}$ & & & $\begin{array}{c}0.6 \\
(0.4-0.9)\end{array}$ & & & & \\
\hline $\begin{array}{l}\text { Diagnosed childhood } \\
\text { ADHD }\end{array}$ & Yes & & & $\begin{array}{c}3 \cdot 1 \\
(1 \cdot 2-8 \cdot 2)\end{array}$ & & & $\begin{array}{c}3.4 \\
(1.8-6 \cdot 2)\end{array}$ & & \\
\hline $\begin{array}{l}\text { Household income } \\
\text { per capita }\end{array}$ & $\begin{array}{l}>=1500 \mathrm{RMB} / \\
\text { month }\end{array}$ & $\begin{array}{c}1.5 \\
(1 \cdot 1-2 \cdot 0)\end{array}$ & & & & $\begin{array}{c}1.4 \\
(1 \cdot 1-1.8)\end{array}$ & $\begin{array}{c}1 \cdot 7 \\
(1 \cdot 1-2 \cdot 4)\end{array}$ & & \\
\hline $\begin{array}{l}\text { Maternal education } \\
\text { level }\end{array}$ & $\begin{array}{l}>=\text { High school } \\
\text { graduate }\end{array}$ & $\begin{array}{c}1.5 \\
(1 \cdot 2-1 \cdot 9)\end{array}$ & & & & & & $\begin{array}{c}2 \cdot 4 \\
(1 \cdot 6-3 \cdot 6)\end{array}$ & \\
\hline $\begin{array}{l}\text { Paternal education } \\
\text { level }\end{array}$ & $\begin{array}{l}>=\text { High school } \\
\text { graduate }\end{array}$ & & & & $\begin{array}{c}1.8 \\
(1 \cdot 2-2 \cdot 7)\end{array}$ & & & & $\begin{array}{c}1.9 \\
(1.4-2.6)\end{array}$ \\
\hline $\begin{array}{l}\text { Paternal education } \\
\text { level *Household } \\
\text { income per capita }\end{array}$ & $\begin{array}{l}>=\text { High school } \\
\text { graduate and }>= \\
\text { 1500RMB } / \text { month }\end{array}$ & & & & & & $\begin{array}{c}1.9 \\
(1.3,2.8)\end{array}$ & & \\
\hline Common cold & $>5$ times/year & $\begin{array}{c}2 \cdot 3 \\
(1 \cdot 8-2 \cdot 8)\end{array}$ & $\begin{array}{c}3 \cdot 2 \\
(2 \cdot 6-4 \cdot 0)\end{array}$ & $\begin{array}{c}3.5 \\
(1.9-6 \cdot 6)\end{array}$ & $\begin{array}{c}2 \cdot 7 \\
(1 \cdot 8-4 \cdot 1)\end{array}$ & $\begin{array}{c}2 \cdot 3 \\
(1 \cdot 7-3 \cdot 1)\end{array}$ & $\begin{array}{c}1.6 \\
(1 \cdot 1-2 \cdot 6)\end{array}$ & & $\begin{array}{c}1 \cdot 7 \\
(1 \cdot 2-2 \cdot 4)\end{array}$ \\
\hline $\begin{array}{l}\text { Diagnosed recurrent } \\
\text { otitis media }\end{array}$ & Yes & $\begin{array}{c}2 \cdot 0 \\
(1 \cdot 3-3 \cdot 0)\end{array}$ & $\begin{array}{c}2 \cdot 3 \\
(1 \cdot 6-3 \cdot 4)\end{array}$ & $\begin{array}{c}5 \cdot 4 \\
(1 \cdot 9-16 \cdot 2)\end{array}$ & $\begin{array}{c}3 \cdot 3 \\
(1 \cdot 7-6 \cdot 7)\end{array}$ & $\begin{array}{c}2.5 \\
(1 \cdot 6-3 \cdot 9)\end{array}$ & $\begin{array}{c}3 \cdot 1 \\
(1 \cdot 6-5 \cdot 8)\end{array}$ & & \\
\hline Snoring & Yes & $\begin{array}{c}1 \cdot 6 \\
(1 \cdot 2-2 \cdot 0)\end{array}$ & $\begin{array}{c}1.9 \\
(1.4-2 \cdot 4)\end{array}$ & & $\begin{array}{c}2 \cdot 4 \\
(1 \cdot 5-3 \cdot 7)\end{array}$ & & $\begin{array}{c}1.7 \\
(1 \cdot 0-2 \cdot 9)\end{array}$ & & \\
\hline $\begin{array}{l}\text { Sleep-disordered } \\
\text { breathing }\end{array}$ & Yes & $\begin{array}{c}2.4 \\
(1.5-3.9)\end{array}$ & & $\begin{array}{c}7.0 \\
(2 \cdot 5-19 \cdot 6)\end{array}$ & $\begin{array}{c}2 \cdot 3 \\
(1 \cdot 1-4 \cdot 6)\end{array}$ & & $\begin{array}{c}2 \cdot 9 \\
(1 \cdot 4-6 \cdot 2)\end{array}$ & $\begin{array}{c}2 \cdot 3 \\
(1 \cdot 1-5 \cdot 1)\end{array}$ & \\
\hline Paternal snoring & Yes & & $\begin{array}{c}1.4 \\
(1 \cdot 1-1 \cdot 8)\end{array}$ & & & & & & $\begin{array}{c}1.4 \\
(1.0-1.9)\end{array}$ \\
\hline Maternal snoring & Yes & & & & & & & $\begin{array}{c}2 \cdot 7 \\
(1 \cdot 3-5 \cdot 6)\end{array}$ & \\
\hline $\begin{array}{l}\text { Parental age at child's } \\
\text { birth }\end{array}$ & & & & & $\begin{array}{c}1 \cdot 1 \\
(1 \cdot 0-1 \cdot 3)\end{array}$ & & & & \\
\hline $\begin{array}{l}\text { Maternal age at } \\
\text { child's birth }\end{array}$ & & $\begin{array}{c}1 \cdot 0 \\
(1 \cdot 0-1 \cdot 1)\end{array}$ & & & & & & & \\
\hline Diagnosed asthma & Yes & $\begin{array}{c}5 \cdot 1 \\
(3 \cdot 9-6 \cdot 8)\end{array}$ & $\begin{array}{c}3.6 \\
(2 \cdot 2-5 \cdot 9)\end{array}$ & & $\begin{array}{c}4.8 \\
(2 \cdot 5-9 \cdot 2)\end{array}$ & $\begin{array}{c}5 \cdot 1 \\
(3 \cdot 4-1 \cdot 7)\end{array}$ & $\begin{array}{c}3 \cdot 3 \\
(1 \cdot 3-8 \cdot 1)\end{array}$ & $\begin{array}{c}6 \cdot 4 \\
(2 \cdot 2-18 \cdot 2)\end{array}$ & $\begin{array}{c}2.9 \\
(1 \cdot 2-6 \cdot 7)\end{array}$ \\
\hline Diagnosed eczema & Yes & $\begin{array}{c}1.5 \\
(1 \cdot 1-2 \cdot 1)\end{array}$ & $\begin{array}{c}1.9 \\
(1.3-2.9)\end{array}$ & & & $\begin{array}{c}2.5 \\
(1.5-4 \cdot 0)\end{array}$ & & $\begin{array}{c}3.9 \\
(2 \cdot 2-6 \cdot 9)\end{array}$ & $\begin{array}{c}1.8 \\
(1 \cdot 1-3 \cdot 1)\end{array}$ \\
\hline $\begin{array}{l}\text { Common } \\
\text { cold*Snoring }\end{array}$ & Yes & $\begin{array}{c}2.4 \\
(1.4,3.9)\end{array}$ & & & & & & & \\
\hline
\end{tabular}

Values highlighted in bold indicate $\mathrm{OR}$ within $95 \% \mathrm{Cl}$ and statistically significant at $\mathrm{P}<0.05$.

Because a stepwise selection procedure was used, only significant variables were included in the final models (Tables 3,4,5).

\section{Discussion}

The present study reveals a geographic variation in the prevalences of allergic diseases across different regions of China. In particular, the regional variation in the prevalence of asthma was even wider than those in the US [8]. In general, the prevalence rates of allergic diseases were higher in more developed areas than poor areas.

The spectrum of risk factors for allergies in China had some common features with that in developed countries.
Firstly, a cluster of unhealthy lifestyle factors which are widespread in developed countries such as diagnosed obesity, frequent use of computer for amusement (indicating physical inactivity) and excessive carbonated drinks intake (indicating unhealthy diets) were also important risk factors of childhood allergies in China [9-11]. Secondly, we found that a series of mental-healthrelated factors such as childhood ADHD, prepartum and postpartum depression, parental depression, non-nuclear family structure including single-parental or extended family and overburdened schoolwork were highly associated with the prevalences of childhood allergic diseases at the individual, household and school level. 
Table 5 Local level risk factors of eczema: results of multiple logistic regression analysis

\begin{tabular}{|c|c|c|c|c|c|c|c|c|c|}
\hline Variables & & $\begin{array}{c}\text { Shanghai } \\
\text { OR } \\
(95 \% \mathrm{Cl})\end{array}$ & $\begin{array}{c}\text { Guangzhou } \\
\text { OR } \\
(95 \% \mathrm{Cl})\end{array}$ & $\begin{array}{l}\text { Xi'an OR } \\
(95 \% \mathrm{Cl})\end{array}$ & $\begin{array}{c}\text { Wuhan } \\
\text { OR } \\
(95 \% \mathrm{Cl})\end{array}$ & $\begin{array}{c}\text { Chengdu } \\
\text { OR } \\
(95 \% \mathrm{Cl})\end{array}$ & $\begin{array}{l}\text { Harbin OR } \\
(95 \% \mathrm{Cl})\end{array}$ & $\begin{array}{c}\text { Hohhot } \\
\text { OR } \\
(95 \% \mathrm{Cl})\end{array}$ & $\begin{array}{c}\text { Urumqi } \\
\text { OR } \\
(95 \% \mathrm{Cl})\end{array}$ \\
\hline Gender & Male & $\begin{array}{c}0.7 \\
(0.5-0.9)\end{array}$ & & & & & & & \\
\hline Diagnosed Obesity & Yes & $\begin{array}{c}2 \cdot 0 \\
(1 \cdot 1-3 \cdot 7)\end{array}$ & & & & & & & \\
\hline \multirow[t]{2}{*}{ Mode of delivery } & $\begin{array}{l}\text { Caesarean } \\
\text { (Ref.) }\end{array}$ & & & & & & & & \\
\hline & Vaginal & & & & & & & $\begin{array}{c}0.6 \\
(0.4-0.7)\end{array}$ & \\
\hline $\begin{array}{l}\text { Carbonated drinks intake } \\
\text { like Coke }\end{array}$ & $\begin{array}{l}>=5 \text { times/ } \\
\text { week }\end{array}$ & & & & & & & $\begin{array}{c}2 \cdot 0 \\
(1 \cdot 1-3 \cdot 1)\end{array}$ & \\
\hline $\begin{array}{l}\text { Computer use as } \\
\text { amusements }\end{array}$ & $\begin{array}{l}>=5 \text { times/ } \\
\text { week }\end{array}$ & & & & & $\begin{array}{c}2 \cdot 0 \\
(1 \cdot 3-3 \cdot 3)\end{array}$ & & $\begin{array}{c}2 \cdot 1 \\
(1 \cdot 2-3 \cdot 9)\end{array}$ & \\
\hline Paternal smoking & Yes & & & $\begin{array}{c}1 \cdot 8 \\
(1 \cdot 0-3 \cdot 1)\end{array}$ & & & & & \\
\hline $\begin{array}{l}\text { Diagnosed childhood } \\
\text { ADHD }\end{array}$ & Yes & & & & $\begin{array}{c}2 \cdot 2 \\
(1 \cdot 2-3 \cdot 9)\end{array}$ & $\begin{array}{c}2 \cdot 7 \\
(1 \cdot 4-5 \cdot 3)\end{array}$ & & & \\
\hline $\begin{array}{l}\text { Paternal diagnosed } \\
\text { depression }\end{array}$ & Yes & & & & $\begin{array}{c}2.7 \\
(1.5-6 \cdot 8)\end{array}$ & & & & \\
\hline $\begin{array}{l}\text { Diagnosed prepartum and } \\
\text { postpartum depression }\end{array}$ & Yes & & $\begin{array}{c}2 \cdot 4 \\
(1 \cdot 1-5 \cdot 8)\end{array}$ & $\begin{array}{c}5 \cdot 9 \\
(2 \cdot 4-14 \cdot 6)\end{array}$ & & & & $\begin{array}{c}4.7 \\
(2 \cdot 0-10 \cdot 8)\end{array}$ & \\
\hline Overburdened schoolwork & Yes & & & & & & & & $\begin{array}{c}2 \cdot 0 \\
(1 \cdot 3-3 \cdot 0)\end{array}$ \\
\hline Maternal education level & $\begin{array}{l}>=\text { High } \\
\text { school } \\
\text { graduate }\end{array}$ & $\begin{array}{c}1.5 \\
(1 \cdot 1-1.9)\end{array}$ & $\begin{array}{c}1 \cdot 6 \\
(1 \cdot 1-2 \cdot 2)\end{array}$ & & & & $\begin{array}{c}1.5 \\
(1 \cdot 1-2 \cdot 1)\end{array}$ & & $\begin{array}{c}2 \cdot 0 \\
(1 \cdot 3-3 \cdot 0)\end{array}$ \\
\hline Paternal education level & $\begin{array}{l}>=\text { High } \\
\text { school } \\
\text { graduate }\end{array}$ & & & & & & & $\begin{array}{c}2 \cdot 1 \\
(1 \cdot 4-3 \cdot 2)\end{array}$ & \\
\hline Common cold & $>5$ times/year & $\begin{array}{c}2.0 \\
(1 \cdot 5-2 \cdot 7)\end{array}$ & & $\begin{array}{c}3 \cdot 7 \\
(2 \cdot 0-6 \cdot 9)\end{array}$ & $\begin{array}{c}2.4 \\
(1.5-3 \cdot 7)\end{array}$ & & $\begin{array}{c}2 \cdot 8 \\
(1 \cdot 9-4 \cdot 1)\end{array}$ & $\begin{array}{c}1 \cdot 8 \\
(1 \cdot 1-3 \cdot 1)\end{array}$ & $\begin{array}{c}3.0 \\
(1.9-4.5)\end{array}$ \\
\hline Snoring & Yes & & $\begin{array}{c}1 \cdot 6 \\
(1 \cdot 1-2 \cdot 3)\end{array}$ & & & & & & \\
\hline Sleep-disordered breathing & Yes & $\begin{array}{c}1 \cdot 8 \\
(1 \cdot 0-3 \cdot 3)\end{array}$ & & & & & & & \\
\hline Paternal snoring & Yes & & & & & & & & $\begin{array}{c}1.7 \\
(1 \cdot 1-2 \cdot 5)\end{array}$ \\
\hline $\begin{array}{l}\text { Diagnosed recurrent otitis } \\
\text { media }\end{array}$ & Yes & $\begin{array}{c}2 \cdot 2 \\
(1 \cdot 4-3 \cdot 6)\end{array}$ & & & & $\begin{array}{c}2 \cdot 1 \\
(1 \cdot 1-4 \cdot 0)\end{array}$ & & & $\begin{array}{c}3.6 \\
(1 \cdot 6-7 \cdot 9)\end{array}$ \\
\hline Diagnosed asthma & Yes & $\begin{array}{c}3 \cdot 1 \\
(2 \cdot 2-4 \cdot 6)\end{array}$ & $\begin{array}{c}2 \cdot 7 \\
(1 \cdot 4-5 \cdot 1)\end{array}$ & $\begin{array}{c}4.0 \\
(1 \cdot 0-15 \cdot 6)\end{array}$ & & $\begin{array}{c}2 \cdot 4 \\
(1 \cdot 3-4 \cdot 4)\end{array}$ & $\begin{array}{c}3.5 \\
(1.5-7.9)\end{array}$ & $\begin{array}{c}6.9 \\
(2.4-2 \cdot 0)\end{array}$ & $\begin{array}{c}3.9 \\
(1 \cdot 6-9 \cdot 6)\end{array}$ \\
\hline Diagnosed allergic rhinitis & Yes & $\begin{array}{c}1.5 \\
(1 \cdot 1-2 \cdot 1)\end{array}$ & $\begin{array}{c}1.9 \\
(1.3-2 \cdot 8)\end{array}$ & & $\begin{array}{c}2 \cdot 1 \\
(1 \cdot 2-3 \cdot 7)\end{array}$ & $\begin{array}{c}2 \cdot 4 \\
(1 \cdot 5-3 \cdot 9)\end{array}$ & $\begin{array}{c}2 \cdot 1 \\
(1 \cdot 1-3 \cdot 8)\end{array}$ & $\begin{array}{c}3 \cdot 2 \\
(1 \cdot 8-5 \cdot 7)\end{array}$ & \\
\hline
\end{tabular}

Values highlighted in bold indicate $\mathrm{OR}$ within $95 \% \mathrm{Cl}$ and statistically significant at $\mathrm{P}<0.05$.

However, some social determinants seem to have played more important roles in the occurrence of asthma in China than in developed countries. For example, prolonged exclusive breast-feeding (OR allergic rhinitis: 0.84; $95 \% \mathrm{CI}: 0.76,0.93)$ and vaginal delivery $\left(\mathrm{OR}_{\text {asthma: }}\right.$ 0.82; 95\% CI: $0.69,0.98)$ were both protective factors for allergies. Nevertheless in China the rates of these factors are markedly low. China's recently updated national rate of "exclusive breast feeding for six months" reached $21.6 \%$, which was far below the WHO target of 100\% [12-14]. On the other hand, a recent WHO survey reveals that
China had the world's highest rate of caesarean sections (up to $46.2 \%$ ), far exceeding the recommended upper threshold by WHO (15\%)[15]. More concerning is that, among all the observed caesarean cases, $25 \%$ were done without medical indications, of which $62 \%$ were caused by financial incentives of hospitals [16].

Another striking difference between China and developed countries is the effects of mental health problems on allergic diseases: the stressors that were often found in developed countries at community or neighborhood level such as frequent exposure to violence and crime 
due to existence of residential segregation were rarely found in China[17]. On the other hand, stressors from the school, such as overburdened schoolwork detected in our study (OR eczema: 1.96; 95\% CI: 1.27, 2.98) (Table $5)$, have become more worrisome in China, since a highly competitive educational system had forced children to be exposed to high levels of stress from the start of primary school, which had caused lots of childhood psychosomatic symptoms [18].

A third difference is the effect of SES represented by parental education and household income per capita. The present study found that higher individual-level SES predicted higher prevalence of allergic diseases in China, in contrast to the findings from the developed countries that higher SES predicted lower prevalence[17], suggesting different hypothesis for explaining the impact of SES on allergies between China and developed countries.

Considering the local pattern of childhood allergies, some specific determinants influenced by social, cultural, and institutional structures were significantly associated with the local prevalence of allergic diseases. For example, psychological risk factors seem to have a considerable impact on the prevalence of asthma in Wuhan (Table 3). In fact, the proportion of childhood ADHD, prepartum and postpartum depression, and parental anxiety and depression in Wuhan were among the highest in the eight cities (Table 3), and all these were major risk factors of allergies. In Chengdu, some factors related to unhealthy dietary habits were found to be key risk factors of allergies, such as diagnosed GER (OR: 14.6; 95\% CI: 12.1-41.7). Chengdu is well-known for its historic food culture featuring heavy-oiled hot pot. It is widely believed that heavy-oiled hot pot is associated with GER, which is a leading risk factor of asthma in Chengdu.

Further investigation of the difference among cities using multi-level models found a significant association between childhood allergies and city-level socio-environmental factors. For example, GDP, PM10 and average humidity were strongly associated with childhood asthma. These will be discussed in a separate paper.

There are several limitations of the present study. Firstly, all the sampling sites were cities, thus only representing urban/suburban areas. Secondly, because this study was initially designed to address the issue of childhood sleep, there were some important allergic risk factors that were not included, for example, family history of asthma and allergies, and indoor and outdoor allergens.

\section{Conclusions}

The present study found strong evidence of the connection between social, behavioural and biological factors and allergic diseases in China, indicating that strategies to reduce exposure to risk factors for childhood allergies could be broadened. In addition, local policies should specifically target at the risk factors for each individual area.

\section{Additional material}

\begin{abstract}
Additional File 1: Figure S1- Location of the sampling cities. The eight cities were: Shanghai, Guangzhou, Xi'an, Wuhan, Harbin, Chengdu, Hohhot and Urumqi. These were capital cities of provinces located in four different regions cited from the 2006 China Statistical Yearbook according to their geographic locations, economic standards, and population densities.

Additional File 2: Figure S2-Samples and the sampling cities. These sampling cities were capital cities of provinces located in four different regions cited from the 2006 China Statistical Yearbook according to their geographic locations, economic standards, and population densities. Three to ten districts were randomly selected from each city, and 1-2 elementary schools from each district. The selection was proportional to size so the number of districts was determined by the sizes of the cities and the number of schools determined by the sizes of the districts. The final sample comprised thirty districts and 42 schools in urban areas, and 9 districts and 13 schools in suburban areas.

Additional File 3: TableS1 - Characteristics of study subjects. A wide range of variables concerning demographic, lifestyle, mental health, socioeconomic status and health conditions were also covered in this table.
\end{abstract}

\section{Acknowledgements}

The work was funded by Ministry of Education of China (NCET program), National Science Foundation of China (81000592, 11001084), Science and Technology Commission of Shanghai Municipality (10DZ2272200, 09DZ2200900, 10PJ1407500, 10PJ1403500, 10231203903 and 10JC1411200), Shanghai Municipal Education Commission (11ZZ103). Shanghai Municipal Health Bureau (2010004) and Morning Star Rewarding Fund (Category B, 2011) of Shanghai Jiaotong University.

\section{Author details}

${ }^{1}$ Department of Developmental and Behavioral Pediatrics, Shanghai Institute of Pediatric Translational Medicine, Shanghai Children's Medical Centre, Shanghai Jiaotong University, School of Medicine, Shanghai, China. ${ }^{2}$ Shanghai Key Laboratory of Children's Environmental Health, Shanghai Jiaotong University, School of Medicine, Shanghai, China. ${ }^{3}$ The Key Laboratory of Children's Environmental Health, Ministry of Education, China. ${ }^{4}$ Department of Statistics and Actuarial Sciences, East China Normal University, Shanghai, China. ${ }^{5}$ School of Public Health and Institute of Health and Biomedical Innovation, Queensland University of Technology, Queensland, 4000, Australia.

\section{Authors' contributions}

This study was planned and implemented by XMS and ST, who are the principal investigators. XMS was responsible for the study's overall conception and design, acquisition of funding, supervision of data collection, and advice on clinical diagnostic methods. ST was responsible for the study's design and advice on epidemiological methods, data interpretation, critical revisions of the manuscript for important intellectual content, FL contributed to the conception and design of the study, analysis and interpretation of data, critical revisions of the manuscript for important intellectual content, and clinical diagnostic expertise for the study. YZ contributed to the analysis and interpretation of data, drafting of the manuscript, and epidemiological expertise for the study. SL, FJ, XJ, CY, YT and $Y Z$ implemented the study and offered critical revisions of the manuscript for important intellectual content. All authors read and approved the final manuscript. 


\section{Competing interests}

The authors declare that they have no competing interests.

Received: 11 February 2011 Accepted: 6 June 2011

Published: 6 June 2011

\section{References}

1. Global burden of asthma. [http://www.ginasthma.org/pdf/ GINABurdenReport.pdf ].

2. Asthma, Chronic respiratory diseases. Quick asthma facts. [http://www. who.int/respiratory/asthma/en/index.html].

3. Asher MI, Montefort S, Björkstén B, Lai CK, Strachan DP, Weiland SK, Williams H, ISAAC Phase Three Study Group: Worldwide time trends in the prevalence of symptoms of asthma, allergic rhino-conjunctivitis, and atopic dermatitis in childhood: ISAAC Phases One and Three repeat multicounty cross-sectional surveys. Lancet 2006, 368:733-743.

4. Chen YZ, National Cooperation Group On Childhood Asthma: Comparative analysis of the state of asthma prevalence in children from two nationwide surveys in 1990 and 2000 year. Zhonghua Jiehe He Huxi Za Zhi 2004, 24:112-116, (in Chinese)..

5. Li SH, Jin XM, Yan CH, Wu SH, Jiang F, Shen XM: Bed- and Room-sharing in Chinese school-aged children: prevalence and association with sleep behaviours. Sleep Medicine 2008, 9:555-563.

6. National Bureau of statistic of China: China Statistical Yearbook 2006 Beijing: China Statistics Press; 2006, (in Chinese).

7. ChanYeung M, Anthonisen NR, Becklake MR, Bowie D, Sonia Buist A Dimich-Ward H, Ernst P, Sears MR, Siersted HC, Sweet L, Van Til L, Manfreda J: Geographical variations in the prevalence of atopic sensitization in six study sites across Canada. Allergy 2010.

8. Child Current Asthma Prevalence Rate (Percent) and Prevalence (Number) by Age and State or Territory. [http://www.cdc.gov/asthma/ brfss/05/child/current/C3.PDF].

9. Wright RJ, Subramanian SV: Advancing a multilevel framework for epidemiologic research on asthma disparities. Chest 2007, 132(Suppl):757-769.

10. Eder W, Ege MJ, von Mutius E: The asthma epidemic. N Engl J Med 2006, 355:2226-2235

11. Kaaks R, Toniolo P, Akhmedkhanov A, Lukanova A, Biessy C, Dechaud H, Dechaud H, Rinaldi S, Zeleniuch-Jacquotte A, Shore RE, Riboli E: Serum Cpeptide, insulin-like growth factor (IGF)-I, IGF-binding proteins, and colorectal cancer risk in women. I Natl Cancer Inst 2000, 92:1592-1600.

12. Government and UN meet to tackle declining breastfeeding rates in China. [http://www.unicef.org/china/media_921.html].

13. National Program of Action for Child Development in China (2000-2010). [http://www.nwccw.gov.cn/html/49/n-140649-2.html].

14. China lagging behind breastfeeding targets. [http://www.chinadaily.com cn/china/2009-08/08/content_8546104.htm].

15. Althabe F, Belizán JM: Caesarean section: the paradox. Lancet 2006, 368:1472-1473.

16. Lumbiganon P, Laopaiboon M, World Health Organization Global Survey on Maternal and Perinatal Health Research Group: Method of delivery and pregnancy outcomes in Asia: the WHO global survey on maternal and prenatal health 2007-08. Lancet 2010, 375:490-499.

17. Williams DR, Sternthal $M$, Wright RJ: Social determinants: taking the social context of asthma seriously. Pediatrics 2009, 123(Suppl):174-184.

18. Xu F, Qiu L, Binns CW, Liu X: Breastfeeding in China: a review. Int Breastfeed J 2009, 16:4-6.

\section{Pre-publication history}

The pre-publication history for this paper can be accessed here: http://www.biomedcentral.com/1471-2458/11/437/prepub

doi:10.1186/1471-2458-11-437

Cite this article as: $L i$ et al:: Prevalence and risk factors of childhood allergic diseases in eight metropolitan cities in China: A multicenter study. BMC Public Health 2011 11:437. 